\title{
Survey of Dental Health Care Workers Regarding Their Knowledge of Viral Liver Disease and Prevention of Its Transmission, Using an Online Questionnaire
} \author{
Shiratsuchi ${ }^{7}$ \\ ${ }^{1}$ Department of Organ System Interactions and Information, Saga University, Japan \\ ${ }^{2}$ Department of Social Dentistry, Nihon University School of Dentistry, Japan \\ ${ }^{3}$ Center for Comprehensive Community Medicine, Saga University, Japan \\ ${ }^{4}$ Division of Disease Control \& Molecular Epidemiology, Health Sciences University of Hokkaido, Japan \\ ${ }^{5}$ Department of Oral and Maxillofacial Surgery, Yamaguchi University Graduate School of Medicine, Japan \\ ${ }^{6}$ Division of Clinical Nursing, School of Nursing, Faculty of Medicine, Yamagata University, Japan \\ ${ }^{7}$ Shiratsuchi Dental Clinic, Japan
}

Yumiko Nagao ${ }^{*}$, Tetsunori Ozaki ${ }^{2}$, Atsushi Kawaguchi ${ }^{3}$, Itsuo Chiba ${ }^{4}$, Koji Harada ${ }^{5}$ Takafumi Saito ${ }^{6}$ and Seiji

Submission: June 27, 2018; Published: September 18, 2018

"Corresponding author: Yumiko Nagao, Department of Organ System Interactions and Information, Faculty of Medicine, Saga University, 5-1-1 Nabeshima, Saga 849-8501, Japan, Telephone: +81-952-34-2516, Fax: +81-952-34-2516; Email: nagaoyu@cc.saga-u.ac.jp

Abstract

Background: Over three million people in Japan are estimated to be infected with hepatitis viruses. Dentists need to be aware of measures to prevent transmission and have knowledge of extrahepatic manifestations. However, in Japan, there has been little evaluation of dentists knowledge of viral hepatitis. We investigated dental care workers for their knowledge of, and countermeasures against, viral hepatitis.

Materials and methods: An anonymous online questionnaire surveying 1,210 members of the Japanese Society of Dental Practice Administration. Interviews were carried out with those who consented. Survey items are attributes, self-management of viral hepatitis, knowledge of liver disease, control of transmission, contacting patients with viral hepatitis, and gathering information on liver diseases.

Results: 153 individuals responded to the questionnaire; 41 had not been immunized against hepatitis B and 61 knew of extrahepatic manifestations. Risk and knowledge deficit scores were significantly higher for workers in dental clinics than those in university settings $(\mathrm{p}<0.001, \mathrm{p}=0.014)$.

Conclusion: The respondents had insufficient knowledge of viral hepatitis, a low rate of immunization against hepatitis B and may not follow safe medical practice. It is critical that dentists understand the latest information on hepatitis viruses and acquire knowledge and skills related to medical safety and prevention of infection.

Keywords: Dental health care workers, Online questionnaire survey, Hepatitis C virus, Hepatitis B virus, Oral lichen planus, Nosocomial infection control

Abbreviations: OLP: Oral Lichen Planus; HCV: Hepatitis C Virus; HBV: Hepatitis B Virus; SVR: Sustained Virological Response; DAA: DirectActing Antivirals

\section{Introduction}

It is estimated that more than 3 million people in Japan are infected with hepatitis B virus (HBV) and/or hepatitis C virus (HCV), and the number of liver cancer deaths annually is about 30,000 [1]. About $65 \%$ of liver cancer is caused by HCV infection and about $15 \%$ is attributable to HBV infection. Recent advances in direct-acting antivirals (DAAs) have been revolutionary in HCV treatment. It is also known that infection with HCV causes damage not only to the liver but also other organs and tissues [2]. Such extrahepatic manifestations include lymphoproliferative, autoimmune, skin-mucosal and metabolic diseases. Lichen 
planus (LP), Sjögren's syndrome, and oral cancer have been reported as extrahepatic manifestations in the oral cavity [3-6].

Japan has been in the forefront of countering hepatitis viruses; the introduction of hepatitis virus tests by the Health Promotion Act (conventional old age health law), the so-called 'turning point examination and examination out of the turning point' (2002), the implementation of free hepatitis examinations (2007), the establishment of the Hepatitis Information Center (2008), the initiation of the medical expenses subsidy program for interferon treatment (2008), and enforcement of the Basic Act on Hepatitis Measures (2010) are of note. Based on the Basic Act on Hepatitis Measures, the underlying philosophy was established, and the responsibilities of the national government and local governments were clarified. Measures against hepatitis have been promoted comprehensively for the prevention, early detection, and treatment of hepatitis.

Meanwhile, because dental care workers often have contact with bodily fluids, such as the blood and saliva of patients, infection control in dental medical institutions is critical. Measures and knowledge of patients infected with HBV or HCV, the most common infections in our country, are essential for dental health workers. However, dental health workers do not necessarily have sufficient knowledge of hepatitis viruses $[7,8]$. Patients with viral hepatitis may be subject to discrimination and prejudice from healthcare workers, and it is also a concern Table 1: Items in the anonymous online questionnaire.

\begin{tabular}{|c|c|c|c|}
\hline Items & No. & Question & Choice \\
\hline \multirow{7}{*}{ Attributes } & 1 & Residential area & $\begin{array}{l}\text { "Hokkaido" “Tohoku” "Kanto" “Chubu” "Kinki” Chugoku” "Shikoku” } \\
\text { "Kyush \& Okinawa” }\end{array}$ \\
\hline & 2 & Sex & "Male" “Female" \\
\hline & 3 & Age & “30s” “40s” “50s" “60s” “70s" "over 80s” \\
\hline & 4 & Institution & $\begin{array}{l}\text { "University / research institution" "General hospital” "Clinic" "Local } \\
\text { public entity" "Company" "Other" }\end{array}$ \\
\hline & 5 & Occupation & $\begin{array}{l}\text { "Dentist" "Doctor" "Dental hygienist" “Dental assistant" "Dental } \\
\text { technician" "Nurse" "Medical clerk" "Pharmacist" "Clinical laboratory } \\
\text { technician" "Radiologist" "Managed dietician" "Public health nurse" } \\
\text { "Administrative staff" "Corporate employee" "Student" "Other" }\end{array}$ \\
\hline & 6 & Affiliated society & "Japanese Society for Oral Health" “Others" \\
\hline & 7 & Years of service & $\begin{array}{c}\text { "Less than } 5 \text { years" " } 5 \text { to } 10 \text { years" "10 to } 20 \text { years" " } 20 \text { years or } \\
\text { more" }\end{array}$ \\
\hline \multirow{5}{*}{$\begin{array}{l}\text { Self-management for } \\
\text { viral hepatitis }\end{array}$} & 8 & Examination of HBV infection & "I have received" "I have not received" "I do not know" \\
\hline & 9 & Examination of HCV infection & "I have received" "I have not received" "I do not know" \\
\hline & 10 & $\begin{array}{l}\text { Presence or absence of immunization } \\
\text { against hepatitis B }\end{array}$ & "I have received" "I have not received" "I do not know" \\
\hline & 11 & $\begin{array}{l}\text { Presence or absence of HBV-related } \\
\text { liver disease }\end{array}$ & $\begin{array}{c}\text { "I am suffering" "I suffered from hepatitis B a long time ago" "I am } \\
\text { not suffering" "I do not know" }\end{array}$ \\
\hline & 12 & $\begin{array}{l}\text { Presence or absence of HCV-related } \\
\text { liver disease }\end{array}$ & $\begin{array}{c}\text { "I am suffering" "I suffered from hepatitis C a long time ago" "I am } \\
\text { not suffering" "I do not know" }\end{array}$ \\
\hline \multirow{2}{*}{$\begin{array}{l}\text { Knowledge of liver } \\
\text { disease }\end{array}$} & 13 & $\begin{array}{l}\text { Knowledge that people infected with } \\
\text { HCV are more common in West Japan }\end{array}$ & "I know well" "I know" "I know little" "I do not know at all" \\
\hline & 14 & $\begin{array}{l}\text { Standard treatment for hepatitis } C \\
\text { using oral antiviral drugs }\end{array}$ & "I know well" "I know" "I know little" "I do not know at all" \\
\hline
\end{tabular}

that they may not declare their own hepatitis virus infection at a dental clinic $[9,10]$.

To date, there have been few reports in Japan where dentists have been investigated in detail regarding their recognition of viral liver disease. Therefore, in this study, we conducted a survey using the Internet to evaluate the recognition of problems of hepatitis by dentists. If we can clarify the problem using this survey, we can contribute to the dissemination of knowledge of liver diseases to dental health workers.

\section{Methods}

\section{Subjects}

The subjects are the 1,210 members of the Japanese Society of Dental Practice Administration.

\section{Methods}

After all members of the Japanese Society of Dental Practice Administration had been notified, we made the anonymous, questionnaire available online from April 27 to June 30, 2017. We explained the purpose of this study on the first page of the online questionnaire and considered those who responded to have provided consent. After answering the anonymous questionnaire survey, those who agreed to a telephone interview provided their name, address and contact telephone number and submitted a written consent form. 


\section{Advanced Research in Gastroenterology \& Hepatology}

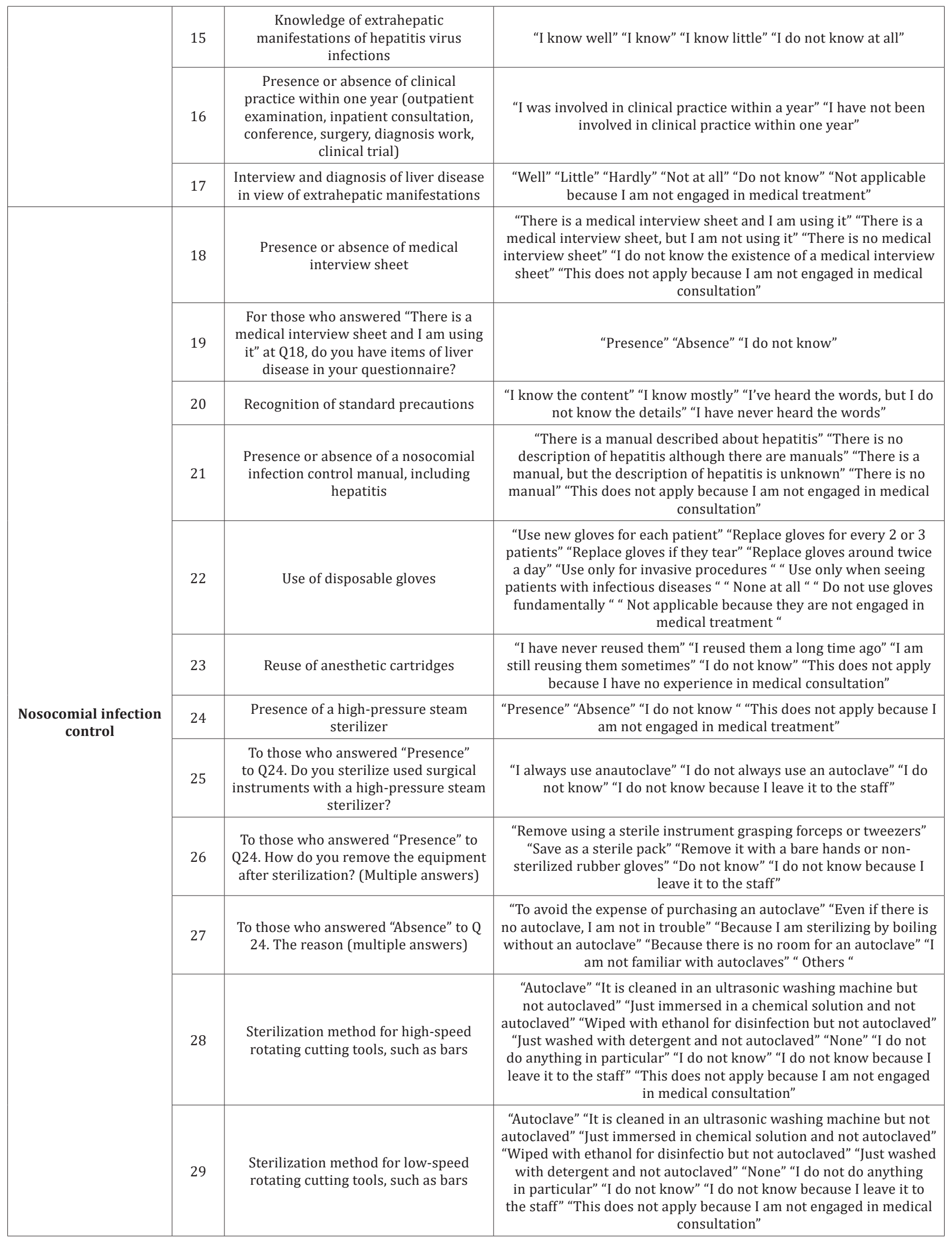




\section{Advanced Research in Gastroenterology \& Hepatology}

\begin{tabular}{|c|c|c|c|}
\hline & 30 & $\begin{array}{l}\text { Management of used high-speed air } \\
\text { turbine handpieces }\end{array}$ & $\begin{array}{l}\text { "Autoclave sterilization"”Wipe with ethanol for disinfection but do } \\
\text { not autoclave"”I do not do anything in particular" "I do not know" } \\
\text { "I do not know because I leave it to the staff" "This does not apply } \\
\text { because I am not engaged in medical treatment" }\end{array}$ \\
\hline & 31 & $\begin{array}{l}\text { To those who answered "autoclave } \\
\text { sterilization" to Q30. Regarding the rate } \\
\text { of exchange }\end{array}$ & $\begin{array}{l}\text { "Always replace after each patient" "Exchange sometimes" "Exchange } \\
\text { after infectious patients" "I do not know" "I do not know because I } \\
\text { leave it to the staff" }\end{array}$ \\
\hline & 32 & $\begin{array}{l}\text { Management of used low-speed contra- } \\
\text { angle handpieces }\end{array}$ & $\begin{array}{l}\text { "Autoclave sterilization" "Wipe with ethanol for disinfection but do } \\
\text { not autoclave"”I do not do anything in particular" "I do not know" } \\
\text { "I do not know because I leave it to the staff" "This does not apply } \\
\text { because I ams not engaged in medical treatment" }\end{array}$ \\
\hline & 33 & $\begin{array}{l}\text { Management of used endodontic } \\
\text { treatment instruments (reamers, files, } \\
\text { cleansers, etc.) }\end{array}$ & $\begin{array}{l}\text { "Autoclave sterilization" "It is cleaned in an ultrasonic washing } \\
\text { machine but not autoclaved" "Just immersed in chemical solution } \\
\text { and not autoclaved" "Wiped with ethanol for disinfection but not } \\
\text { autoclaved" "Just washed with detergent and not autoclaved" } \\
\text { "Nothing" "I do not do anything in particular" "I do not know" "I } \\
\text { do not know because I leave it to the staff" "This does not apply } \\
\text { because I am not engaged in medical treatment" }\end{array}$ \\
\hline & 34 & $\begin{array}{l}\text { Disinfection method for rubber or } \\
\text { plastic equipment used for hepatitis } \\
\text { virus infected persons }\end{array}$ & $\begin{array}{l}\text { "Immerse in glutaral or phthalal preparations" "Wipe with ethanol } \\
\text { for disinfection" "Because it cannot be put in an autoclave, it is often } \\
\text { discarded after use" "Only wash with detergent" "Nothing" "Do not } \\
\text { know" "I do not know because I leave it to the staff" "This does not } \\
\text { apply because I am not engaged in medical treatment" }\end{array}$ \\
\hline \multirow{4}{*}{$\begin{array}{l}\text { How to contact } \\
\text { patients with viral } \\
\text { hepatitis }\end{array}$} & 35 & $\begin{array}{l}\text { Experience of having problems with } \\
\text { hepatitis virus patients }\end{array}$ & $\begin{array}{l}\text { "Frequently" "Occasionally" "Very little" "Not at all" "Do not know" } \\
\text { "Not applicable because I am not engaged in medical treatment" }\end{array}$ \\
\hline & 36 & $\begin{array}{l}\text { To those who answered "Frequently" or } \\
\text { "Occasionally" when a person infected } \\
\text { with hepatitis virus was consulted in } \\
\text { Q 35. The most troublesome (multiple } \\
\text { answers) }\end{array}$ & $\begin{array}{l}\text { "Infection control against liver diseases" "Understanding the } \\
\text { pathology of liver diseases" "How to treat liver diseases and nursing" } \\
\text { "Observation treatment (tooth extraction and incision, etc.)" } \\
\text { "Medication" "Communication with patients" "Doctor's introduction } \\
\text { and inquiries to the doctor" "Communication with a doctor } \\
\text { specializing in liver disease" "Others" }\end{array}$ \\
\hline & 37 & $\begin{array}{l}\text { To those who answered "Frequently" or } \\
\text { "Occasionally when a person infected } \\
\text { with hepatitis virus was consulted in } \\
\text { Q35, The most troublesome (single } \\
\text { answers) }\end{array}$ & $\begin{array}{l}\text { Infection control against liver diseases "Understanding the } \\
\text { pathology of liver diseases" "How to treat liver diseases and nursing" } \\
\text { "Observation treatment (tooth extraction and incision, etc.)" } \\
\text { "Medication" "Communication with patients" "Doctor's introduction } \\
\text { and inquiries to the doctor" "Communication with a doctor } \\
\text { specializing in liver disease" "Others" }\end{array}$ \\
\hline & 38 & $\begin{array}{l}\text { Prejudice and discrimination against } \\
\text { hepatitis virus infected patients }\end{array}$ & "Much" "A little" "Not so much" "No at all” "Do not know" \\
\hline $\begin{array}{l}\text { Method of gathering } \\
\text { information on liver } \\
\text { diseases }\end{array}$ & 39 & $\begin{array}{l}\text { Do you want to participate in a seminar } \\
\text { that provides the latest information on } \\
\text { hepatitis? }\end{array}$ & $\begin{array}{l}\text { "I want to participate" "I do not want to participate too much" "Will } \\
\text { not participate" "I do not know" }\end{array}$ \\
\hline Telephone interview & 40 & Do you agree to a telephone interview? & "I do not agree" "I agree" \\
\hline
\end{tabular}

HBV: Hepatitis B Virus; HCV: Hepatitis C Virus

\section{Items in the anonymous, online questionnaire (Table 1):}

a) Attributes: Residential area, sex, age, institution, occupation, affiliated society, and years of service

b) Self-management of viral hepatitis: Examination of HBV infection, examination of HCV infection, whether immunized against hepatitis B, presence or absence of HBV-related liver disease, and presence or absence of HCV-related liver disease

c) Knowledge of liver disease: Knowledge that infection with HCV is more common in West Japan, standard treatment for hepatitis $\mathrm{C}$, knowledge of extrahepatic manifestations of infection with hepatitis viruses, and interview and diagnosis of liver disease in view of such extrahepatic manifestations

d) Nosocomial infection control: Presence or absence of medical interview forms, recognition of standard precautions, presence or absence of a nosocomial infection control manual, use of disposable gloves, reuse of anesthetic cartridges, presence of a high-pressure steam sterilizer, and method of sterilization of cutting tools, rotary cutting machines and instruments for root canal treatment after use

e) How to contact patients with viral hepatitis.

f) Method of gathering information on liver diseases.

Analysis of items regarding risk behavior and onset of liver disease, and lack of knowledge (Table 2): Among the questionnaire items, risk scores were given to responses that indicated failure to self-manage hepatitis or to take safe hospital infection countermeasures. Risk was scored as 1 point and norisk as zero point. In addition, a responder with liver disease was scored as 1 point and one without liver disease as zero point. A person with no knowledge of liver disease was scored as one 
point and one with knowledge was scored as zero point. For each subject, the scores were totaled for three items: risk behavior, presence of liver disease and lack of knowledge. A high score indicates a dangerous medical situation.

Table 2: Result of online questionnaire by anonymity (153 people).

\begin{tabular}{|c|c|c|c|c|c|c|c|c|}
\hline Items & No. & & Variable & $\mathbf{n}$ & $\%$ & $\begin{array}{l}\text { Score of Risk } \\
\text { Behavior }\end{array}$ & $\begin{array}{l}\text { Score of Liver } \\
\text { Disease Onset }\end{array}$ & $\begin{array}{c}\text { Score of Lack } \\
\text { Knowledge }\end{array}$ \\
\hline \multirow{31}{*}{ Attributes } & \multirow{8}{*}{1} & \multirow{8}{*}{ Residential area } & Hokkaido & 4 & $2.6 \%$ & & & \\
\hline & & & Tohoku & 12 & $7.8 \%$ & & & \\
\hline & & & Kanto & 49 & $32.0 \%$ & & & \\
\hline & & & Chubu & 19 & $12.4 \%$ & & & \\
\hline & & & Kinki & 14 & $9.2 \%$ & & & \\
\hline & & & Chugoku & 17 & $11.1 \%$ & & & \\
\hline & & & Shikoku & 7 & $4.6 \%$ & & & \\
\hline & & & Kyush \& Okinawa & 31 & $20.3 \%$ & & & \\
\hline & \multirow{2}{*}{2} & \multirow{2}{*}{ Sex } & Male & 128 & $83.7 \%$ & & & \\
\hline & & & Female & 25 & $16.3 \%$ & & & \\
\hline & \multirow{5}{*}{3} & \multirow{5}{*}{ Age } & $30 \mathrm{~s}$ & 10 & $6.5 \%$ & & & \\
\hline & & & $40 \mathrm{~s}$ & 25 & $16.3 \%$ & & & \\
\hline & & & $50 s$ & 62 & $40.5 \%$ & & & \\
\hline & & & $60 \mathrm{~s}$ & 51 & $33.3 \%$ & & & \\
\hline & & & $70 \mathrm{~s}$ & 5 & $3.3 \%$ & & & \\
\hline & \multirow{5}{*}{4} & \multirow{5}{*}{ Institution } & $\begin{array}{l}\text { University / research } \\
\text { institution }\end{array}$ & 46 & $30.1 \%$ & & & \\
\hline & & & General hospital & 4 & $2.6 \%$ & & & \\
\hline & & & Clinic & 99 & $64.7 \%$ & & & \\
\hline & & & Company & 1 & $0.7 \%$ & & & \\
\hline & & & Other & 3 & $2.0 \%$ & & & \\
\hline & \multirow{5}{*}{5} & \multirow{5}{*}{ Occupation } & Dentist & 140 & $91.5 \%$ & & & \\
\hline & & & Dental hygienist & 10 & $6.5 \%$ & & & \\
\hline & & & Dental technician & 1 & $0.7 \%$ & & & \\
\hline & & & Pharmacist & 1 & $0.7 \%$ & & & \\
\hline & & & Corporate employee & 1 & $0.7 \%$ & & & \\
\hline & \multirow[t]{2}{*}{6} & \multirow[t]{2}{*}{ Affiliated society } & $\begin{array}{c}\text { Japanese Society for Oral } \\
\text { Health }\end{array}$ & 45 & $29.4 \%$ & & & \\
\hline & & & Others & 120 & $78.4 \%$ & & & \\
\hline & \multirow{4}{*}{7} & \multirow{4}{*}{ Years of service } & Less than 5 years & 2 & $1.3 \%$ & & & \\
\hline & & & 5 to 10 years & 6 & $3.9 \%$ & & & \\
\hline & & & 10 to 20 years & 26 & $17.0 \%$ & & & \\
\hline & & & 20 years or more & 119 & $77.8 \%$ & & & \\
\hline \multirow{10}{*}{$\begin{array}{c}\text { Self- } \\
\text { manage- } \\
\text { ment } \\
\text { of viral } \\
\text { hepatitis }\end{array}$} & \multirow{3}{*}{8} & \multirow{3}{*}{$\begin{array}{l}\text { Examination of } \mathrm{HBV} \\
\text { infection }\end{array}$} & I have received & 144 & $94.1 \%$ & 0 & & \\
\hline & & & I have not received & 8 & $5.2 \%$ & 1 & & \\
\hline & & & I do not know & 1 & $0.7 \%$ & 1 & & \\
\hline & \multirow{3}{*}{9} & \multirow{3}{*}{$\begin{array}{c}\text { Examination of HCV } \\
\text { infection }\end{array}$} & I have received & 126 & $82.4 \%$ & 0 & & \\
\hline & & & I have not received & 24 & $15.7 \%$ & 1 & & \\
\hline & & & I do not know & 3 & $2.0 \%$ & 1 & & \\
\hline & \multirow{3}{*}{10} & \multirow{3}{*}{$\begin{array}{c}\text { Presence or absence of } \\
\text { immunization against } \\
\text { hepatitis B }\end{array}$} & I have received & 109 & $71.2 \%$ & 0 & & \\
\hline & & & I have not received & 41 & $26.8 \%$ & 1 & & \\
\hline & & & I do not know & 3 & $2.0 \%$ & 1 & & \\
\hline & 11 & $\begin{array}{c}\text { Presence or absence of } \\
\text { own HBV-related liver } \\
\text { disease }\end{array}$ & I am suffering & 2 & $1.3 \%$ & & 1 & \\
\hline
\end{tabular}


Advanced Research in Gastroenterology \& Hepatology

\begin{tabular}{|c|c|c|c|c|c|c|c|c|}
\hline & & & $\begin{array}{l}\text { I suffered from hepatitis B a } \\
\text { long time ago }\end{array}$ & 2 & $1.3 \%$ & & 1 & \\
\hline & & & I am not suffering & 148 & $96.7 \%$ & & 0 & \\
\hline & & & I do not know & 1 & $0.7 \%$ & & 0 & \\
\hline & & & I am suffering & 1 & $0.7 \%$ & & 1 & \\
\hline & 12 & $\begin{array}{l}\text { Presence or absence of } \\
\text { own HCV-related liver }\end{array}$ & $\begin{array}{l}\text { I suffered from hepatitis } \mathrm{C} \mathrm{a} \\
\text { long time ago }\end{array}$ & 1 & $0.7 \%$ & & 1 & \\
\hline & & disease & I am not suffering & 150 & $98.0 \%$ & & 0 & \\
\hline & & & I do not know & 1 & $0.7 \%$ & & 0 & \\
\hline \multirow{20}{*}{$\begin{array}{l}\text { Knowledge } \\
\text { of liver } \\
\text { disease }\end{array}$} & \multirow{4}{*}{13} & \multirow{4}{*}{$\begin{array}{l}\text { Knowledge that more } \\
\text { people are infected with } \\
\text { HCV are more common in } \\
\text { West Japan }\end{array}$} & I know well & 21 & $13.7 \%$ & & & 0 \\
\hline & & & I know & 51 & $33.3 \%$ & & & 0 \\
\hline & & & I know little & 56 & $36.6 \%$ & & & 1 \\
\hline & & & I do not know at all & 25 & $16.3 \%$ & & & 1 \\
\hline & \multirow{4}{*}{14} & \multirow{4}{*}{$\begin{array}{l}\text { Standard treatment for } \\
\text { hepatitis } C \text { using oral } \\
\text { antiviral drugs }\end{array}$} & I know well & 28 & $18.3 \%$ & & & 0 \\
\hline & & & I know & 86 & $56.2 \%$ & & & 0 \\
\hline & & & I know little & 25 & $16.3 \%$ & & & 1 \\
\hline & & & I do not know at all & 14 & $9.2 \%$ & & & 1 \\
\hline & \multirow{4}{*}{15} & \multirow{4}{*}{$\begin{array}{c}\text { Knowledge of } \\
\text { extrahepatic } \\
\text { manifestations of } \\
\text { hepatitis virus infections }\end{array}$} & I know well & 8 & $5.2 \%$ & & & 0 \\
\hline & & & I know & 53 & $34.6 \%$ & & & 0 \\
\hline & & & I know little & 73 & $47.7 \%$ & & & 1 \\
\hline & & & I do not know at all & 19 & $12.4 \%$ & & & 1 \\
\hline & \multirow{8}{*}{17} & \multirow{2}{*}{$\begin{array}{l}\text { Presence or absence of } \\
\text { clinical practice within } \\
\text { one year (outpatient } \\
\text { examination, inpatient } \\
\text { consultation, conference, } \\
\text { surgery, diagnosis work, } \\
\text { clinical trial) }\end{array}$} & $\begin{array}{l}\text { I was involved in clinical } \\
\text { practice within a year }\end{array}$ & 125 & $81.7 \%$ & & & \\
\hline & & & $\begin{array}{l}\text { I have not been involved in } \\
\text { clinical practice within one } \\
\text { year }\end{array}$ & 28 & $18.3 \%$ & & & \\
\hline & & \multirow{6}{*}{$\begin{array}{l}\text { Interview and diagnosis } \\
\text { of liver disease in } \\
\text { view of extrahepatic } \\
\text { manifestations }\end{array}$} & Well & 12 & $7.8 \%$ & & & 0 \\
\hline & & & Little & 22 & $14.4 \%$ & & & 0 \\
\hline & & & Hardly & 47 & $30.7 \%$ & & & 1 \\
\hline & & & Not at all & 48 & $31.4 \%$ & & & 1 \\
\hline & & & Do not know & 4 & $2.6 \%$ & & & 1 \\
\hline & & & $\begin{array}{l}\text { Not applicable because it } \\
\text { is not engaged in medical } \\
\text { treatment }\end{array}$ & 20 & $13.1 \%$ & & & \\
\hline \multirow{10}{*}{$\begin{array}{l}\text { Nosocomial } \\
\text { infection } \\
\text { control }\end{array}$} & \multirow{3}{*}{18} & \multirow{3}{*}{$\begin{array}{l}\text { Presence or absence of } \\
\text { medical interview sheet }\end{array}$} & $\begin{array}{l}\text { There is a medical interview } \\
\text { sheet and I am using it }\end{array}$ & 139 & $90.8 \%$ & 0 & & \\
\hline & & & $\begin{array}{l}\text { There is no medical } \\
\text { interview sheet }\end{array}$ & 1 & $0.7 \%$ & 1 & & \\
\hline & & & $\begin{array}{l}\text { It does not apply because } \\
\text { it is not engaged in medical } \\
\text { consultation }\end{array}$ & 13 & $8.5 \%$ & 0 & & \\
\hline & \multirow{4}{*}{19} & \multirow{4}{*}{$\begin{array}{l}\text { For those who answered } \\
\text { "There is a medical } \\
\text { interview sheet and I am } \\
\text { using it" at Q18, do you } \\
\text { have items of liver disease } \\
\text { in your questionnaire? }\end{array}$} & $\begin{array}{l}\text { Those who do not answer } \\
\text { "medical interview sheet } \\
\text { exists and uses" in Q18" }\end{array}$ & 14 & $9.2 \%$ & & & \\
\hline & & & Presence & 128 & $83.7 \%$ & 0 & & \\
\hline & & & Absence & 10 & $6.5 \%$ & 1 & & \\
\hline & & & I do not know & 1 & $0.7 \%$ & 1 & & \\
\hline & \multirow{3}{*}{20} & \multirow{3}{*}{$\begin{array}{l}\text { Recognition of standard } \\
\text { precautions }\end{array}$} & I know the content & 97 & $63.4 \%$ & 0 & & \\
\hline & & & I know mostly & 29 & $19.0 \%$ & 0 & & \\
\hline & & & $\begin{array}{l}\text { I've heard the words but I do } \\
\text { not know the details }\end{array}$ & 19 & $12.4 \%$ & 1 & & \\
\hline
\end{tabular}


Advanced Research in Gastroenterology \& Hepatology

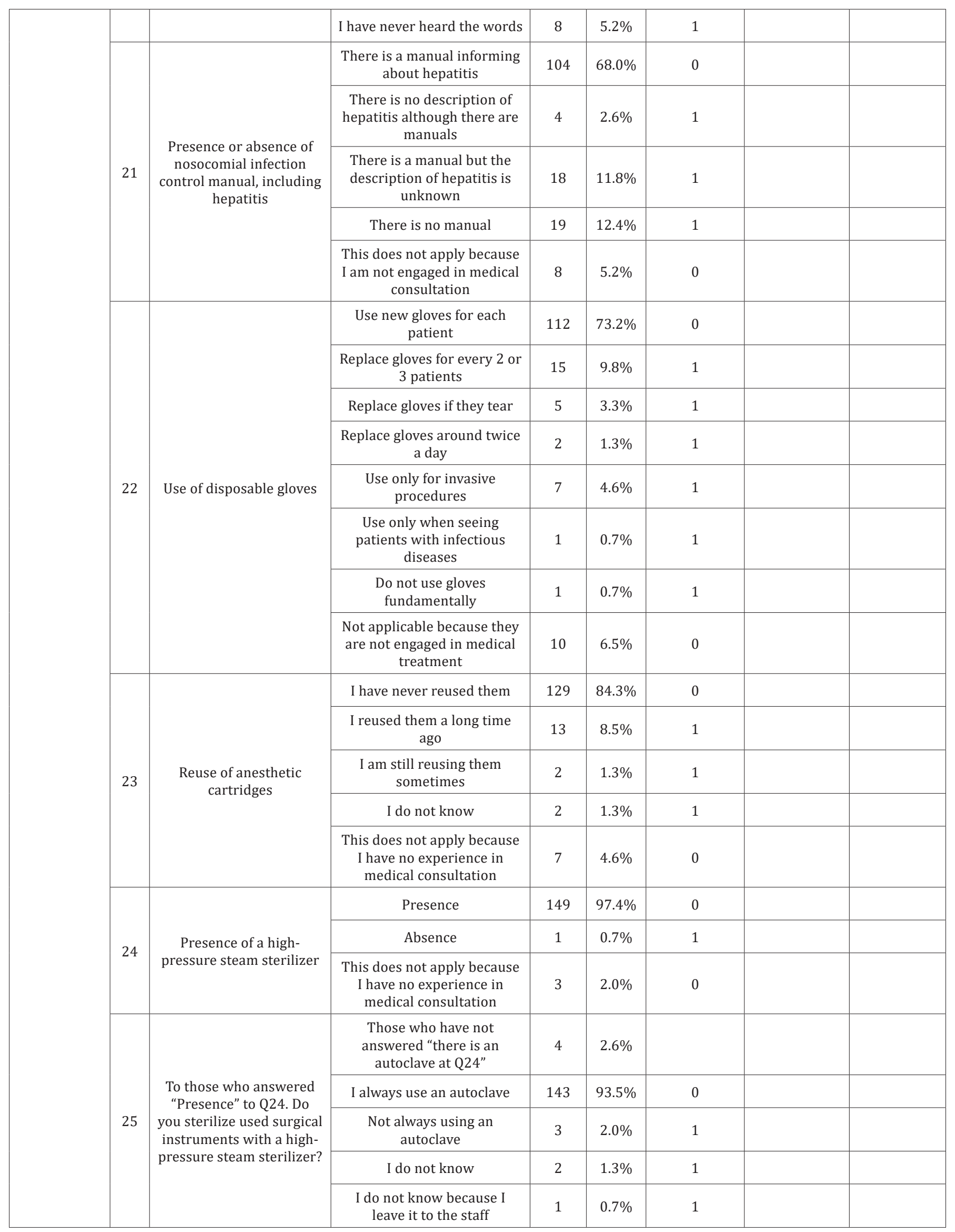




\begin{tabular}{|c|c|c|c|c|c|}
\hline \multirow{6}{*}{26} & \multirow{6}{*}{$\begin{array}{l}\text { To those who answered } \\
\text { "Presence" to Q24. } \\
\text { How do you remove } \\
\text { the equipment after } \\
\text { sterilization? (Multiple } \\
\text { answers) }\end{array}$} & $\begin{array}{c}\text { Remove using a sterile } \\
\text { instrument grasping forceps } \\
\text { or tweezers }\end{array}$ & 75 & $49.0 \%$ & 0 \\
\hline & & Save as a sterile pack & 95 & $62.1 \%$ & 0 \\
\hline & & $\begin{array}{l}\text { Remove it with a bare hand } \\
\text { or non-sterilized rubber } \\
\text { glove }\end{array}$ & 12 & $7.8 \%$ & 1 \\
\hline & & Do not know & 1 & $0.7 \%$ & 1 \\
\hline & & $\begin{array}{l}\text { I do not know because I } \\
\text { leave it to the staff }\end{array}$ & 8 & $5.2 \%$ & 1 \\
\hline & & Other & 1 & $0.7 \%$ & 0 \\
\hline 27 & $\begin{array}{l}\text { To those who answered } \\
\text { "Absence" to Q24. } \\
\text { The reason (multiple } \\
\text { answers) }\end{array}$ & Other & 1 & $0.7 \%$ & \\
\hline \multirow{8}{*}{28} & \multirow{8}{*}{$\begin{array}{c}\text { Sterilization method } \\
\text { for high-speed rotating } \\
\text { cutting tools, such as bars }\end{array}$} & Autoclave & 106 & $69.3 \%$ & 0 \\
\hline & & $\begin{array}{l}\text { This does not apply because } \\
\text { I have no experience in } \\
\text { medical consultation }\end{array}$ & 11 & $7.2 \%$ & 0 \\
\hline & & $\begin{array}{c}\text { It is cleaned in an ultrasonic } \\
\text { washing machine but not } \\
\text { autoclaved }\end{array}$ & 14 & $9.2 \%$ & 1 \\
\hline & & $\begin{array}{l}\text { Just immersed in a chemical } \\
\text { solution and not autoclaved }\end{array}$ & 10 & $6.5 \%$ & 1 \\
\hline & & $\begin{array}{c}\text { Wiped with ethanol } \\
\text { for disinfection but not } \\
\text { autoclaved }\end{array}$ & 3 & $2.0 \%$ & 1 \\
\hline & & None & 4 & $2.6 \%$ & 1 \\
\hline & & I do not know & 3 & $2.0 \%$ & 1 \\
\hline & & $\begin{array}{l}\text { I do not know because I } \\
\text { leave it to the staff }\end{array}$ & 2 & $1.3 \%$ & 1 \\
\hline \multirow{9}{*}{29} & \multirow{9}{*}{$\begin{array}{c}\text { Sterilization method } \\
\text { for low-speed rotating } \\
\text { cutting tools, such as bars }\end{array}$} & Autoclave & 87 & $56.9 \%$ & 0 \\
\hline & & $\begin{array}{l}\text { This does not apply because } \\
\text { I have no experience in } \\
\text { medical consultation }\end{array}$ & 10 & $6.5 \%$ & 0 \\
\hline & & $\begin{array}{l}\text { It is cleaned in an ultrasonic } \\
\text { washing machine but not } \\
\text { autoclaved }\end{array}$ & 19 & $12.4 \%$ & 1 \\
\hline & & $\begin{array}{l}\text { Just immersed in a chemical } \\
\text { solution but not autoclaved }\end{array}$ & 18 & $11.8 \%$ & 1 \\
\hline & & $\begin{array}{l}\text { Wiped with ethanol } \\
\text { for disinfection but not } \\
\text { autoclaved }\end{array}$ & 6 & $3.9 \%$ & 1 \\
\hline & & Nothing & 7 & $4.6 \%$ & 1 \\
\hline & & $\begin{array}{l}\text { I do not do anything in } \\
\text { particular }\end{array}$ & 2 & $1.3 \%$ & 1 \\
\hline & & I do not know & 2 & $1.3 \%$ & 1 \\
\hline & & $\begin{array}{c}\text { I do not know because I } \\
\text { leave it to the staff }\end{array}$ & 2 & $1.3 \%$ & 1 \\
\hline \multirow{4}{*}{30} & \multirow{4}{*}{$\begin{array}{l}\text { Management of used } \\
\text { high-speed air turbine } \\
\text { handpieces }\end{array}$} & Autoclave sterilization & 118 & $77.1 \%$ & 0 \\
\hline & & $\begin{array}{l}\text { Wiped with ethanol } \\
\text { for disinfection but not } \\
\text { autoclaved }\end{array}$ & 20 & $13.1 \%$ & 1 \\
\hline & & I do not know & 1 & $0.7 \%$ & 1 \\
\hline & & $\begin{array}{l}\text { I do not know because I } \\
\text { leave it to the staff }\end{array}$ & 2 & $1.3 \%$ & 1 \\
\hline
\end{tabular}


Advanced Research in Gastroenterology \& Hepatology

\begin{tabular}{|c|c|c|c|c|c|c|c|}
\hline & & & $\begin{array}{l}\text { This does not apply because } \\
\text { I have no experience in } \\
\text { medical consultation }\end{array}$ & 12 & $7.8 \%$ & 0 & \\
\hline & \multirow{4}{*}{31} & \multirow{4}{*}{$\begin{array}{l}\text { To those who answered } \\
\text { "autoclave sterilization" } \\
\text { to Q30. Regarding the rate } \\
\text { of exchange }\end{array}$} & $\begin{array}{l}\text { Those who did not answer } \\
\text { "to autoclave" in Q30 }\end{array}$ & 35 & $22.9 \%$ & & \\
\hline & & & $\begin{array}{c}\text { Always replace after each } \\
\text { patient }\end{array}$ & 102 & $66.7 \%$ & 0 & \\
\hline & & & Exchange sometimes & 11 & $7.2 \%$ & 1 & \\
\hline & & & $\begin{array}{l}\text { Exchange after infectious } \\
\text { patients }\end{array}$ & 5 & $3.3 \%$ & 1 & \\
\hline & \multirow{5}{*}{32} & \multirow{5}{*}{$\begin{array}{l}\text { Management of used } \\
\text { low-speed contra-angle } \\
\text { handpieces }\end{array}$} & Autoclave sterilization & 107 & $69.9 \%$ & 0 & \\
\hline & & & $\begin{array}{l}\text { Wipe with ethanol for } \\
\text { disinfection but do not } \\
\text { autoclave }\end{array}$ & 32 & $20.9 \%$ & 1 & \\
\hline & & & I do not know & 1 & $0.7 \%$ & 1 & \\
\hline & & & $\begin{array}{l}\text { I do not know because I } \\
\text { leave it to the staff }\end{array}$ & 3 & $2.0 \%$ & 1 & \\
\hline & & & $\begin{array}{l}\text { This does not apply because } \\
\text { I have no experience in } \\
\text { medical consultation }\end{array}$ & 10 & $6.5 \%$ & 0 & \\
\hline & \multirow{6}{*}{33} & \multirow{6}{*}{$\begin{array}{l}\text { Management of used } \\
\text { endodontic treatment } \\
\text { instruments (reamers, } \\
\text { files, cleansers, etc.) }\end{array}$} & Autoclave sterilization & 97 & $63.4 \%$ & 0 & \\
\hline & & & $\begin{array}{l}\text { This does not apply because } \\
\text { I have no experience in } \\
\text { medical consultation }\end{array}$ & 14 & $9.2 \%$ & 0 & \\
\hline & & & $\begin{array}{l}\text { It is cleaned in an ultrasonic } \\
\text { washing machine but not } \\
\text { autoclaved }\end{array}$ & 21 & $13.7 \%$ & 1 & \\
\hline & & & $\begin{array}{l}\text { Just immersed ina chemical } \\
\text { solution and not autoclaved }\end{array}$ & 14 & $9.2 \%$ & 1 & \\
\hline & & & Nothing & 5 & $3.3 \%$ & 1 & \\
\hline & & & I do not know & 2 & $1.3 \%$ & 1 & \\
\hline & \multirow{7}{*}{34} & \multirow{7}{*}{$\begin{array}{l}\text { Disinfection method } \\
\text { for rubber or plastic } \\
\text { equipment used for } \\
\text { hepatitis virus infected } \\
\text { persons }\end{array}$} & $\begin{array}{l}\text { Immerse in glutaral or } \\
\text { phthalal preparations }\end{array}$ & 95 & $62.1 \%$ & 0 & \\
\hline & & & $\begin{array}{l}\text { Wipe with ethanol for } \\
\text { disinfection }\end{array}$ & 2 & $1.3 \%$ & 1 & \\
\hline & & & $\begin{array}{l}\text { Because it can not be put } \\
\text { in an autoclave, it is often } \\
\text { discarded after use }\end{array}$ & 35 & $22.9 \%$ & 0 & \\
\hline & & & Nothing & 5 & $3.3 \%$ & 1 & \\
\hline & & & I do not know & 2 & $1.3 \%$ & 1 & \\
\hline & & & $\begin{array}{l}\text { I do not know because I } \\
\text { leave it to the staff }\end{array}$ & 2 & $1.3 \%$ & 1 & \\
\hline & & & $\begin{array}{l}\text { This does not apply because } \\
\text { I have no experience in } \\
\text { medical consultation }\end{array}$ & 12 & $7.8 \%$ & 0 & \\
\hline \multirow{6}{*}{$\begin{array}{l}\text { How to } \\
\text { contact } \\
\text { patients } \\
\text { with viral } \\
\text { hepatitis }\end{array}$} & \multirow{6}{*}{35} & \multirow{6}{*}{$\begin{array}{l}\text { Experience of having } \\
\text { problems with hepatitis } \\
\text { virus patients }\end{array}$} & Frequently & 4 & $2.6 \%$ & & 1 \\
\hline & & & Occasionally & 31 & $20.3 \%$ & & 1 \\
\hline & & & Very little & 84 & $54.9 \%$ & & 0 \\
\hline & & & Not at all & 22 & $14.4 \%$ & & 0 \\
\hline & & & I do not know & 1 & $0.7 \%$ & & 0 \\
\hline & & & $\begin{array}{l}\text { This does not apply because } \\
\text { I have no experience in } \\
\text { medical consultation }\end{array}$ & 11 & $7.2 \%$ & & 0 \\
\hline
\end{tabular}




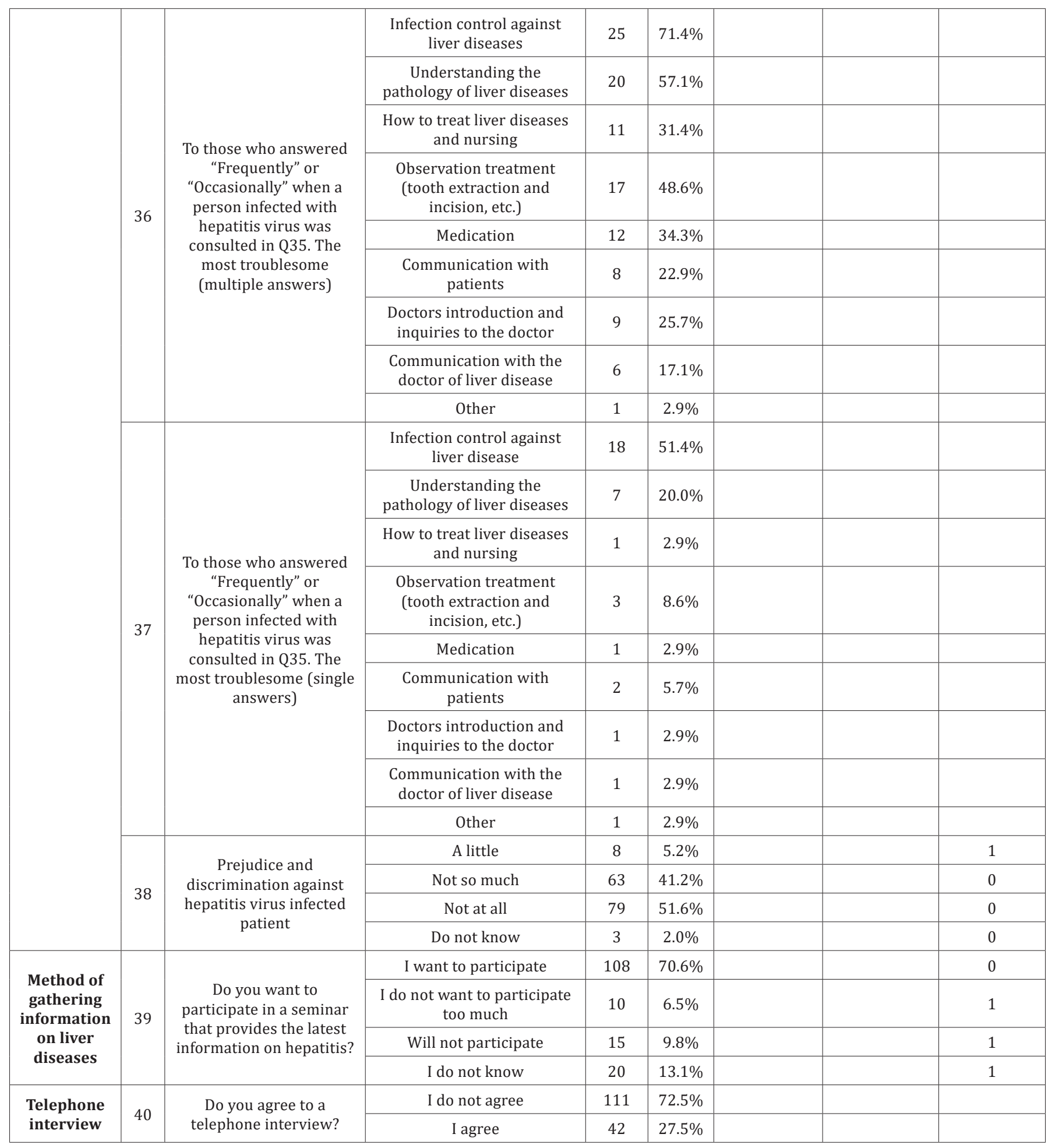

HBV: Hepatitis B Virus; HCV: Hepatitis C Virus

Items of the telephone interviews: We interviewed by telephone those who provided their documented agreement regarding how to cooperate with medical institutions regarding liver disease patients and how to acquire knowledge of liver disease.

Ethics approval and consent to participate: The study was approved by the Ethics Committee of Saga University (reference

number: 28-35 and 29-81) in accordance with the Declaration of Helsinki.

Statistical analysis: Summary statistics are shown as frequencies (proportions) for categorical data and as the mean \pm standard deviation (SD) for continuous variables. The differences between groups were evaluated by the 2-sided unpaired Student's t-test. A p value $<0.05$ was considered statistically significant. All 
statistical analyses were performed using R Statistical Software (version 3.4.3; R Foundation for Statistical Computing, Vienna, Austria).

Results

\section{Aggregate online survey}

153 subjects (128 men, 25 women) completed the online questionnaires anonymously (Table 2). Individuals aged in their fifties $(62,40.5 \%)$ and sixties $(51,33.3 \%)$ accounted for about $70 \%$ of the total. There were more responses from directors of dental clinics (99 individuals, 64.7\%) than university research institutes and workers in general hospitals (50 individuals, $32.7 \%)$. Almost all (91.5\%) of the respondents were dentists. $77.8 \%$ (119 individuals) who had been engaged in medical treatment for more than 20 years.

Of the responders, 144 (94.1\%) and 126 (82.4\%) had been tested for HBV and HCV infection, respectively, but 41 (26.8\%) had not been immunized against hepatitis B. Four $(2.6 \%)$ and two $(1.3 \%)$ had HBV-related and HCV-related liver disease, respectively. Only 72 individuals (47.1\%) were aware that there are more HCV-infected people in the western part of Japan but $114(74.5 \%)$ recognized that oral antiviral agents (direct acting antivirals, DAAs) are the standard therapeutic agents for hepatitis C. Sixty one (39.9\%) knew of the extrahepatic manifestations of hepatitis virus infections and 34 (22.2\%) asked questions and carried out medical examinations regarding HCV infection when examining patients with LP.

The answers to the questions regarding measures for infection control were as follows: There were 10 responders $(6.5 \%)$ who used questionnaires without liver disease items, 126 (82.4\%) who understood the contents of the standard precautions, 104 who had produced manuals for infection control measures, $112(73.2 \%)$ who used disposable gloves for each patient, 129
(84.3\%) who had never recycled anesthetic cartridges, 143 (93.5\%) who sterilized used surgical instruments, 106 (69.3\%) who sterilized used high-speed rotating cutting tools such as bars, 87 (56.9\%) who sterilized used low-speed rotating cutting tools such as bars, 118 (77.1\%) who sterilized used high-speed air turbine handpieces,107 (69.9\%) who sterilized used lowspeed contra-angle handpieces, 97 (63.4\%) who sterilized used endodontic treatment instruments (reamers, etc.), and $130(85.0 \%)$ who disinfected in a safe way or discarded the instruments used for hepatitis virus-infected patients.

The responses regarding the treatment of patients with liver disease were as follows: Thirty-five $(22.9 \%)$ had had difficult experiences with hepatitis virus-infected patients, the most serious problem was infection control against liver disease (18/35 individuals, $51.4 \%$ ), and eight (5.2\%) had prejudice and discrimination against hepatitis virus-infected patients. There were 108 responders $(70.6 \%)$ who wished to participate in seminars providing information on liver diseases.

\section{Analysis of survey items about risk behavior, onset of liver disease, and lack of knowledge}

Table 2 shows the scores for risk behavior, onset of liver disease, and lack of knowledge. Table 3 shows average values of the three sets of scores according to residential district, sex, years of service, and institution. The men had a significantly higher risk scores and knowledge deficit scores than the women $(\mathrm{p}=0.002, \mathrm{p}=0.031)$. Risk scores and knowledge deficit scores were significantly higher for directors of dental clinics than for university workers $(p<0.001, p=0.014)$. Dentists with less than 20 years of experience tended to have a higher knowledge deficit score than those with more than 20 years $(p=0.053)$. There was no significant difference in the relationship between the responder's residential district and the scores.

Table 3: Scores of risk behavior, onset of liver disease, and lack of knowledge.

\begin{tabular}{|c|c|c|c|c|c|}
\hline Residential Districts & Total Score & & West Japan & East Japan & p Value \\
\hline & & & $n=69$ & $n=84$ & \\
\hline & Risk behavior & mean (SD) & $2.99(2.49)$ & $2.98(2.53)$ & 0.982 \\
\hline & Onset of liver disease & mean (SD) & $0.03(0.24)$ & $0.05(0.26)$ & 0.652 \\
\hline & Lack of knowledge & mean (SD) & $2.45(1.29)$ & $2.74(1.55)$ & 0.219 \\
\hline \multirow[t]{5}{*}{ Sex } & & & Male & Female & $p$ value \\
\hline & & & $n=128$ & $\mathrm{n}=25$ & \\
\hline & Risk behavior & mean (SD) & $3.25(2.60)$ & $1.60(1.26)$ & 0.002 \\
\hline & Onset of liver disease & mean (SD) & $0.05(0.28)$ & $0.00(0.00)$ & 0.399 \\
\hline & Lack of knowledge & mean (SD) & $2.72(1.43)$ & $2.04(1.40)$ & 0.031 \\
\hline \multirow[t]{5}{*}{ Years of service } & & & 20 years or more & Less than 20 years & p value \\
\hline & & & $n=119$ & $n=34$ & \\
\hline & Risk behavior & mean (SD) & $2.91(2.42)$ & $3.24(2.80)$ & 0.502 \\
\hline & Onset of liver disease & mean (SD) & $0.04(0.27)$ & $0.03(0.17)$ & 0.799 \\
\hline & Lack of knowledge & mean (SD) & $2.49(1.46)$ & $3.03(1.31)$ & 0.053 \\
\hline
\end{tabular}




\section{Advanced Research in Gastroenterology \& Hepatology}

\begin{tabular}{|l|c|c|c|c|c|}
\hline Institution & & & Dental clinics & $\begin{array}{c}\text { University research institutes and } \\
\text { general hospitals }\end{array}$ & p value \\
\hline & & & $\mathbf{n = 9 9}$ & $1.91(1.98)$ & $<4$ \\
\hline & Risk behavior & mean (SD) & $3.57(2.57)$ & $0.02(0.14)$ & $<0.001$ \\
\hline & Onset of liver disease & mean (SD) & $0.05(0.30)$ & $2.22(1.34)$ & 0.457 \\
\hline & Lack of knowledge & mean (SD) & $2.82(1.46)$ & 0.014 \\
\hline
\end{tabular}

SD: standard deviation

\section{Result of the telephone interviews}

All but one of the 42 subjects who agreed to do so participated in a telephone interview (implementation rate 97.6\%) (Table 4). The average interview time was 14.41 minutes. Twenty-two interviewees (53.7\%) reported "not performing" or "hardly performing" medical cooperation with patients with liver disease. Documents such as letters were the most frequent means of medical cooperation (16 subjects, $39.0 \%$ ). Twenty-five (61.0\%) responded that there were opportunities to acquire knowledge of liver diseases but reported that seminars for dentists about liver diseases were not available.

Table 4: Results of 41 people who underwent a telephone interview.

\begin{tabular}{|c|c|c|c|c|}
\hline Characteristics & & & (n) & $\%$ \\
\hline Sex (n) & & Male/Female & $36 / 5$ & \\
\hline \multirow{5}{*}{ Age (yr) } & & $30 \mathrm{~s}$ & 2 & 4.9 \\
\hline & & $40 \mathrm{~s}$ & 7 & 17.1 \\
\hline & & $50 \mathrm{~s}$ & 15 & 36.6 \\
\hline & & $60 \mathrm{~s}$ & 14 & 34.1 \\
\hline & & $70 \mathrm{~s}$ & 3 & 7.3 \\
\hline Hearing time (minutes) & & Average time & 14.41 & \\
\hline \multirow{4}{*}{ Interview Q1 } & \multirow{4}{*}{$\begin{array}{l}\text { Do you cooperate medically } \\
\text { regarding patients with liver } \\
\text { disease? }\end{array}$} & Yes & 17 & 41.5 \\
\hline & & Almost never & 2 & 4.9 \\
\hline & & Never & 20 & 48.8 \\
\hline & & Not applicable & 2 & 4.9 \\
\hline \multirow{5}{*}{ Interview Q2 } & \multirow{5}{*}{$\begin{array}{l}\text { What is the means of medical } \\
\text { collaboration? (multiple answers) }\end{array}$} & Documents such as letters & 16 & 39.0 \\
\hline & & Telephone & 3 & 7.3 \\
\hline & & Communication via the patient & 2 & 4.9 \\
\hline & & Using a medical cooperation net surg system & 1 & 2.4 \\
\hline & & Not applicable & 1 & 2.4 \\
\hline \multirow{2}{*}{ Interview Q3 } & \multirow{2}{*}{$\begin{array}{l}\text { Do you have the opportunity to } \\
\text { acquire knowledge of liver disease? }\end{array}$} & Yes & 25 & 61.0 \\
\hline & & No & 16 & 39.0 \\
\hline \multirow{4}{*}{ Interview Q4 } & \multirow{4}{*}{$\begin{array}{l}\text { To the } 25 \text { people who answered } \\
\text { Yes to Q3. What is the means } \\
\text { of acquiring knowledge of liver } \\
\text { disease? (Multiple answers) }\end{array}$} & $\begin{array}{l}\text { Participation in academic societies and } \\
\text { lectures }\end{array}$ & 15 & 60.0 \\
\hline & & Reading a book & 9 & 36.0 \\
\hline & & TV programs and newspapers & 1 & 4.0 \\
\hline & & Other & 3 & 12.0 \\
\hline
\end{tabular}

\section{Discussion}

On December 31, 2016, 104,533 dentists were registered in Japan, 80,189 men (76.7\%), and 24,344 women (23.3\%). (Eighty dentists per 100,000 population.) Regarding the type of facility, 89,166 dentists worked in clinics and 12,385 dentists worked in university research institutes and general hospitals: the proportion of dentists working in clinics is increasing year by year.

In this study, the directors of dental clinics had significantly higher risk scores for infection and deficiency of knowledge of hepatitis viruses and infection control than dental physicians working in university research institutes and general hospitals. This indicates a serious problem in securing medical safety in Japan, where the vast majority of dentists work in clinics. Dentists and dental health care workers are at high risk of infection with HBV and HCV during their daily occupational experiences [11].

Following a survey of 253 Japanese dental students between 2006 and 2007, we reported that their understanding of disinfection and sterilization was insufficient [7]. The introduction of a modified curriculum, with appropriate education of students in these matters, were critical issues. 
According to a screening study of 141 Japanese dental workers conducted in 2007 , fewer than half $(48.2 \%)$ of the participants had been immunized against hepatitis B [8]. Of 63 immunized individuals, $16(25.4 \%)$ were positive for anti-HBc, indicating past exposure to the virus. The positivity rate of anti-HBc was $85.7 \%$ for respondents in their sixties and $100 \%$ for those in their seventies; this rate was extremely high for the oldest responders. In routine dental practice, dentists who did not always use disposable gloves accounted for $17 \%$ of those who tested positive. Tada et al. surveyed changes in infection control practice reported by dentists in Japan in 2008 and 2011 and factors related to these changes [12]. They reported that the rate of immunization against hepatitis B was $65.4 \%$ in 2008 and $67.1 \%$ in 2011 . Infection control practices significantly associated with the proportion of dentists specializing in oral surgery, the proportion of dentists reporting a willingness to treat HIV and AIDS patients, and the proportion of dentists reporting knowledge on standard precautions. Compliance with effective infection control practices by dentists may be affected by knowledge and education.

We have also reported that $59.8 \%$ of HBV and HCV infection with liver disease patients consistently self-declared hepatitis virus infection when undergoing dental treatment [9]. The main reason for not reporting such infections at a dental clinic was because the dentist had not enquired about the possibility of an underlying disease $(71.2 \%)$.

In the United States, a case of HBV infection related to tooth extraction was reported in 2007 [13]. Nosocomial infection from patient to patient was proved because the HBV nucleotide sequence matched between the patients. In 2013, in the United States, the Centers for Disease Control and Prevention (CDC) issued a report of patient-to-patient transmission of HCV in a dental office [14]. More than 7,000 patients were notified and tested for hepatitis B and hepatitis $\mathrm{C}$ viruses and HIV because of unsanitary conditions and improper sterilization of equipment in the office. The Oklahoma State Department of Health reported that 77 people tested positive for hepatitis C, five for hepatitis B and four for HIV.

In Japan, Ogata et al. [15] reported that the major sources of acute hepatitis $\mathrm{C}$ virus infection in 2013 were medical procedures and accidental needle sticks. Their study was a retrospective analysis of patients in 12 facilities nationwide who developed acute hepatitis C after 1990. Medical procedures were the most common source of infection, accounting for $32.4 \%$ of the 102 patients (33/102). These procedures were as follows: surgery (14 cases), blood transfusion (5), endoscopy (3), intravenous injection (4), invasive procedures (3), dental therapy (3) and dialysis (1).

Mahboobi et al. [16] concluded in their review that dental treatment is a risk factor for acquiring HBV and HCV and that the risk could be eliminated easily using standard precautionary measures.
Based on a revision of the medical law in Japan, general dental clinics were obliged in 2007 to establish medical safety management systems. In June 2014, the Ministry of Health, Labor and Welfare announced a requirement to sterilize the dental handpieces for each patient. In a questionnaire answered by 700 Japanese dental physicians in 2017, the rate of sterilization after replacing used handpieces for each patient was $52 \%$, the rate of exchanging gloves for each patient was $52 \%$, the rate of sterilization after washing used cutting bars was $64 \%$ and the ratio of sterilization after washing used root canal treatment devices was $65 \%$ [17].

Unfortunately, even in this study of members of the Japanese Society of Dental Practice Administration, the rate of sterilization of instruments was rather low. Despite being at high occupational risk of hepatitis virus infection, only around 70\% of the responders had been immunized against hepatitis B The percentage of dentists who treat oral mucosal disease from the viewpoint of extrahepatic manifestations was also small.

Hepatitis C virus is known to cause extrahepatic manifestations such as oral lichen planus (OLP) $[6,18]$. We performed a genome-wide association study (GWAS) of Japanese HCV-related patients with or without OLP, followed by a replication analysis in an Italian population. It was found that rs884000 in neuropilin-2 (NRP2), rs538399 on insulin-like growth binding proteins factor 4 (IGFBP4), and rs9461799 (HLA-DR/DQ) were associated with HCV-positive LP [19].

If dentists encourage examination and treatment of hepatitis through dental and medical cooperation, they can identify and treat patients with undiagnosed hepatitis virus infections. We examined retrospectively oral mucosal disease and HCV infection using the medical record information of 90 patients who consulted a general dental clinic [20]. OLP was the most common oral disease. Among 51 patients who could be examined for the presence or absence of HCV infection, the incidence of that infection was $29.4 \%(15 / 51)$. Among the OLP patients who consulted the dental clinic, we identified a new HCV-infected patient and led an untreated HCV-infected patient to a sustained virological response (SVR).

There have been some reports that have assessed stigma and discrimination in relation to HCV infection within the healthcare setting [21]. We performed a survey of the prejudice and discrimination experienced by HCV/HBV-infected individuals from healthcare workers. Prejudice was most prevalent within the dental clinic setting [10].

\section{Conclusion}

We conclude that dentists in Japan do not have sufficient knowledge of viral hepatitis, have a low rate of immunization against hepatitis B and do not necessarily carry out safe medical practices, despite having a high risk of infection. We consider that dental care workers need to understand the latest information on hepatitis viruses and to acquire knowledge and skills related 
to medical safety and prevention of infection. It is necessary to treat the oral cavity with a view to cooperative medical treatment between medical departments and dentistry.

\section{Acknowledgement}

We thank Mr. Yuji Kawahigashi (Saga University) for technical support of survey and Dr. Michio Sata (Kurume University School of Medicine, and Nishinihon Hospital) for advice.

\section{Funding}

This study was supported in part by a Grant-in-Aid for Scientific Research (C) (No.17K12012) from the Ministry of Education, Culture, Sports, Science and Technology of Japan.

\section{Authors' Contributions}

YN conceived the study, analyzed and interpreted the data, and was a major contributor in writing the manuscript. IC, $\mathrm{KH}$, and TS contributed greatly to the design of the study. TO and SS conducted data collection. AK conducted statistical analysis and data analysis. SS gave the final approval to be published. All authors read and approved the manuscript.

\section{Competing Interests}

Yumiko Nagao (Corresponding author) belongs to a donationfunded Department funded by Okuda Internal Medicine, Circulatory Medicine and Naniwamarukaiji Inc.. The remaining authors disclose no conflicts.

\section{References}

1. Uemura M, Sasaki Y, Yamada T, Gotoh K, Eguchi H, et al. (2014) Serum antibody titers against hepatitis $\mathrm{C}$ virus and postoperative intrahepatic recurrence of hepatocellular carcinoma. Ann Surg Oncol 21(5): 17191725.

2. Gumber SC, Chopra S (1995) Hepatitis C: a multifaceted disease. Review of extrahepatic manifestations. Ann Intern Med 123(8): 615-620.

3. Nagao Y, Sata M, Tanikawa K, Itoh K, Kameyama T (1995) High prevalence of hepatitis $\mathrm{C}$ virus antibody and RNA in patients with oral cancer. J Oral Pathol Med 24(8): 354-360.

4. Koike K, Moriya K, Ishibashi K, Yotsuyanagi H, Shintani Y, et al. (1997) Sialadenitis histologically resembling Sjögren syndrome in mice transgenic for hepatitis $\mathrm{C}$ virus envelope genes. Proc Natl Acad Sci USA 94(1): 233-236

5. Nagao Y, Hanada S, Shishido S, Ide T, Kumashiro R, et al. (2003) Incidence of Sjögren's syndrome in Japanese patients with hepatitis C virus infection. J Gastroenterol Hepatol 18(3): 258-266.
6. Nagao Y, Sata M (2004) Hepatitis C virus and lichen planus. J Gastroenterol Hepatol 19(10): 1101-1113.

7. Nagao Y, Chiba I, Sata M (2004) Survey of hepatitis B and C in students of faculty of dentistry and dental hygienist school. Kansenshogaku Zasshi 78(7): 554-565.

8. Nagao Y, Matsuoka H, Kawaguchi T, Ide T, Sata M (2008) HBV and HCV infection in Japanese dental care workers. Int J Mol Med 21(6): 791799.

9. Nagao Y, Kawaguchi T, Ide T, Sata M (2008) HCV or HBV infection selfdisclosure to dentists. Kansenshogaku Zasshi 82(3): 213-219.

10. Nagao Y, Kawahigashi Y, Kimura K, Nobayashi H, Sata M (2017) Awareness survey of prejudice and discrimination in hepatitis B and C virus- infected individuals. Adv Res Gastroentero Hepatol 7(1): 1-6.

11. Stewardson DA, Palenik CJ, McHugh ES, Burke FJ (2002) Occupational exposures occurring in students in a UK dental school. Eur J Dent Educ 6(3): 104-113.

12. Tada A, Watanabe M, Senpuku H (2015) Factors affecting changes in compliance with infection control practices by dentists in Japan. Am J Infect Control 43(1): 95-97.

13. Redd JT, Baumbach J, Kohn W, Nainan O, Khristova M, et al. (2007) Patient-to-patient transmission of hepatitis B virus associated with oral surgery. J Infect Dis 195(9): 1311-1314.

14. Bradley KK (2013) Dental healthcare-associated transmission of hepatitis C: final report of public health investigation and response. Oklahoma State Department of Health: Tulsa Health Department.

15. Ogata K, Ide T, Kumashiro R, Kumada H, Yotsuyanagi H, et al. (2006) Timing of interferon therapy and sources of infection in patients with acute hepatitis C. Hepatol Res 34(1): 35-40.

16. Mahboobi N, Porter SR, Karayiannis P, Alavian SM (2013) Dental treatment as a risk factor for hepatitis $\mathrm{B}$ and $\mathrm{C}$ viral infection. A review of the recent literature. J Gastrointestin Liver Dis 22(1): 79-86.

17. Egusa H (2017) Study on development of water purification system for dental unit water supply system (in Japanese). Health Labour Sciences Research. pp. 1-105.

18. Carrozzo M, Scally K (2014) Oral manifestations of hepatitis C virus infection. World J Gastroenterol 20(24): 7534-7543.

19. Nagao Y, Nishida N, Toyo-Oka L, Kawaguchi A, Amoroso A, et al. (2017) Genome-wide association study identifies risk variants for lichen planus in patients with hepatitis C virus infection. Clin Gastroenterol Hepatol 15(6): 937-944.

20. Nagao Y, Tsuji M (2017) The discovery through dentistry of potentially HCV-infected Japanese patients and intervention with treatment. Adv Res Gastroentero Hepatol 7(3): 1-7.

21. Crofts N, Louie R, Loff B (1997) The next plague: stigmatization and discrimination related to hepatitis $\mathrm{C}$ virus infection in Australia. Health Hum Rights 2(2): 87-97.

\begin{tabular}{l} 
Your next submission with JuniperPublishers \\
will reach you the below assets \\
- Quality Editorial service \\
- Swift Peer Review \\
- Reprints availability \\
- E-prints Service \\
- Manuscript Podcast for convenient understanding \\
- Global attainment for your research \\
- Manuscript accessibility in different formats \\
( Pdf, E-pub, Full Text, audio) \\
- Unceasing customer service \\
Track the below URL for one-step submission \\
https://juniperpublishers.com/online-submission.php \\
\hline
\end{tabular}

